\title{
Diseño de robot pez bio inspirado impulsado mediante aleta caudal con 1 GDL.
}

\section{Design of a bioinspired robot driven through caudal fin with 1 DOF.}

Fausto Ramiro Cabrera Aguayo ${ }^{1}$, Fabricio Javier Santacruz Sulca ${ }^{2}$, Byron Hernán Bermeo Jiménez. ${ }^{3} \&$ Cristina Alejandra Orozco Cazco. ${ }^{4}$

Recibido: 10-06-2019 / Revisado: 15-07-2019 /Aceptado: 14-08-2019/ Publicado: 06-09-2019

\begin{abstract}
.
DOI: https://doi.org/10.33262/cienciadigital.v3i3.3.826

This article describes the development of a robotic fish. To comply with caudal propulsion, a 1 DOF (Degrees of freedom) system was designed. In order to test the functionality, it was necessary to seal and proof the electric devices, the design of pieces was developed in Solid Works and printed in 3D in a Prusa I3 Ephestos. Once the robot fish was built a series of underwater tests were applied to check if its movement is equal to its biological similar. The principal problems were due to the tightness and stability, therefore, the robot had to be reassembled a twice of times, finally the prototype swam using caudal propulsion with the body completely submerged in the water.
\end{abstract}

Keywords: Robotics, Bioinspired robots, caudal fin locomotion, underwater vehicles.

\section{Resumen.}

Este articulo describe el desarrollo de un pez robot. Para cumplir con la propulsión caudal, un sistema de 1 GDL (Grados de libertad) fue diseñado. Para probar la funcionalidad, fue necesario sellar e impermeabilizar los dispositivos eléctricos, el

\footnotetext{
${ }^{1}$ Escuela Superior Politécnica de Chimborazo, Facultad de Informática y Electrónica. Riobamba, Ecuador. fausto.cabrera@espoch.edu.ec.

2 Escuela Superior Politécnica de Chimborazo, Facultad de Informática y Electrónica. Riobamba, Ecuador. fabricio.santacruz@espoch.edu.ec.

${ }^{3}$ Instituto Superior Tecnológico Carlos Cisneros. Riobamba, Ecuador. byronbermeo88@ hotmail.com

${ }^{4}$ Instituto Superior Tecnológico Carlos Cisneros. Riobamba, Ecuador. cristymoc@yahoo.es
} 
diseño de piezas fue realizado en Solid Works e impreso en 3D en una prusa I3 Ephestos. Una vez el pez fue construido, una serie de pruebas bajo el agua fueron aplicadas para determinar si la locomoción del robot pez es igual al de su símil biológico. Los problemas principales fueron debido a la impermeabilización y estabilidad, por lo tanto, el robot tuvo que ser reensamblado un par de veces, finalmente el prototipo nadó usando la propulsión por aleta caudal con su cuerpo completamente sumergido en el agua.

Palabras claves: Robótica, Robots Bio-inspirados, locomoción por aleta caudal, vehículos subacuáticos.

\section{Introducción.}

Se conoce que dos de las terceras partes de la superficie de la Tierra están cubiertas por agua. Los océanos permiten el transporte de productos y materia prima entre países, representan fuentes críticas de alimentos y otros recursos como los son el petróleo y el gas, y tienen un gran efecto en el clima y el medio ambiente. El conocimiento científico de los mares profundos está creciendo rápidamente mediante el uso de una variedad de tecnologías. Las primeras exploraciones científicas se llevaron a cabo mediante vehículos submarinos ocupados por humanos. Recientemente, los robots submarinos han comenzado a revolucionar la exploración del fondo marino, ofreciendo generalmente mejor información a un costo más reducido.(Rodríguez Moreno and Cárdenas Martínez 2014)

La robótica bioinspirada se basa en la investigación y simulación de la morfología de los animales, parte de esas investigaciones en robots submarinos se han enfocado en la eficiencia y maniobrabilidad de los peces en el agua, permitiendo el desarrollo de sistemas que ejecutan tareas como mantenimiento e inspección de tuberías de petróleo, aceite en plataformas oceánicas o de gas, como también la explotación geofísica del suelo marino para investigación. En virtud de la capacidad de propulsión híbrida en el cuerpo más la aleta caudal y la maniobrabilidad complementaria en las aletas accesorias, se propone un esquema de propulsión sintetizada que involucra una aleta caudal y un par de aletas pectorales.(Shoele and Zhu 2010) 
Las diferentes aletas, en principio, tienen diferentes funciones, que se complementan entre sí, el cuerpo más la aleta caudal son responsables de la propulsión y la dirección. Las aletas pectorales sirven para el movimiento de las pectorales, La aleta pélvica es extremadamente útil para equilibrar los peces para propulsión, dirección y remo. (Sfakiotakis, Lane, and Davies 1999)

Actualmente se está trabajando constantemente el campo de los peces robóticos, la robótica está involucrada en el estudio de la dinámica de los peces y su control. Se han ideado varias innovaciones en lo que respecta a la mímica del movimiento ondulatorio de los peces, ajustando su movimiento a las curvas apropiada. (Drucker 2006)

Teóricamente hablando, se desea un número infinito de servos de volumen de trabajo infinitamente pequeño para imitar realmente la acción muscular que causa la ondulación de los peces. (Lauder and Madden 2007)

El atún, también es conocido por su nombre científico de Thunnus, comprende alrededor de unas 12 especies que habitan en el océano, este animal posee grandes características en el nado y se conoce como una de las especies más consumidas en las gastronomías del mundo. Aunque posee variadas propiedades que conllevan beneficios en la salud del ser humano, su incremento en la pesca podría significar su extinción como especie, una de las características del atún es que estos animales pueden adaptarse a las temperaturas que brinde el entorno, por ejemplo en el caso de la especie de atún con mayor tamaño conocida como el atún de aleta azul tiene más posibilidades de sobrevivir ante los cambios de hábitats con relación a otras especies de atún, la razón de esto se debe a que cuando las temperaturas del agua se ponen más frías, el atún aleta azul por lo general incrementa su temperatura corporal interna a fin de adaptarse con mayor facilidad.

\section{Metodología.}

La aleta caudal es una aleta impar situada al final del cuerpo del pez, es la aleta de la cola. La aleta caudal puede tener un solo lóbulo o dos lóbulos, es el principal órgano para generar 
el empuje por el que se mueven la mayoría de las especies de peces, normalmente es la más desarrollada y tiene una gran variedad de formas. Tiene dos funciones, por un lado, permite impulsar al pez hacia a delante y, por la otra, actúa como timón para poder girar. La forma específica por la que un pez nada corresponde con la aleta caudal (yu, Liu, and Tan 2008).

La aleta que determina la forma específica por la que un pez nada corresponde con la aleta caudal. La forma de esta aleta o cola trasera del pez aumenta o disminuye la fuerza de empuje de un pez. La aleta caudal del pez atún es fuerte con forma de arco terminado en dos zonas puntiagudas que le dan aspecto de media luna como se observa en la figura 1. (Wang, Alequin-Ramos, and Tan 2011).

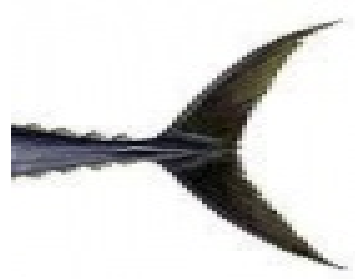

Figura 1. Aleta caudal del pez atún

El flujo de agua alrededor de la aleta caudal es muy importante debido a que permite analizar fácilmente el flujo siguiendo los movimientos de la superficie de la aleta .El método de análisis de flujo se aplicó al análisis del flujo de agua producido por los movimientos de una aleta de la cola de un robot pez. (Villoslada 2016)

Los peces presentan una forma aerodinámica, en virtud de la capacidad de propulsión híbrida en el cuerpo más la aleta caudal y la maniobrabilidad complementaria en las aletas accesorias, se propone un esquema de propulsión sintetizada que involucra una aleta caudal y un par de aletas pectorales. Se han implementado algunos buenos diseños para accionar la parte posterior del cuerpo mediante el constante funcionamiento de motores con un diseño electrónico que le permite simular el movimiento de aleteo que le permitirá desplazarse por el agua

CINEMÁTICA DEL ROBOT

$$
\begin{array}{lr}
x 1=l c 1 \cos q 1 & x \dot{1}==-l c 1 q \dot{1} \sin q 1 \\
y 1=l c 2 \sin q 1 & y \dot{1}=l c 2 q \dot{1} \cos q 1
\end{array}
$$




$$
\begin{aligned}
& \frac{\partial x 2}{\partial q^{2}}=-l c 2 q 2 \sin (q 1+q 2) \\
& \frac{\partial y 2}{\partial q 2}=l c 2 q 2 \cos (q 1+q 2)
\end{aligned}
$$

Primero se desarrollará un análisis cinemático del pez, refiriéndose a ciertos ángulos dados y los parámetros de los elementos del sistema para encontrar las posiciones y orientaciones deseadas de los elementos. Al desarrollar el modelo matemático se inició con el planteamiento para obtener la posición final, de acuerdo al movimiento de cada uno de los eslabones del robot. A continuación, se muestra la cinemática diferencial directa para el robot.

$$
\begin{aligned}
& J^{2} v=\left[\begin{array}{cc}
-l c 1 \sin q 1 & 0 \\
l c 1 \cos q 1 & 0 \\
0 & 0
\end{array}\right]\left[\begin{array}{c}
q 1 \\
\dot{q} 2 \\
0
\end{array}\right] \\
& J^{2} v=\left[\begin{array}{cc}
-l c 1 \sin q 1-l c 2 \sin (q 1+q 2) & -l c 2 \sin (q 1+q 2) \\
l 1 \cos q 1+l c 2 \cos (q 1+q 2) & l c 2 \cos (q 1+q 2) \\
0 & 0
\end{array}\right]\left[\begin{array}{c}
q \dot{1} \\
\dot{q} 2 \\
0
\end{array}\right] \\
& J^{1} w=\left[\begin{array}{ll}
0 & 0 \\
0 & 0 \\
1 & 0
\end{array}\right] \\
& M 1 J^{1} v^{T} J^{1} v \\
& M 1\left[\begin{array}{ccc}
-l c 1 \sin q 1 & l c 1 \cos q 1 & 0 \\
0 & 0 & 0
\end{array}\right]\left[\begin{array}{cc}
-l c 1 \sin q 1 & 0 \\
l c 1 \cos q 1 & 0 \\
0 & 0
\end{array}\right] \\
& M 1=\left[\begin{array}{cc}
M 1 l c 1^{2} & 0 \\
0 & 0
\end{array}\right]
\end{aligned}
$$




$$
J^{1} W^{T} R 1
$$

$$
\left[\begin{array}{lll}
0 & 0 & 1 \\
0 & 0 & 0
\end{array}\right]\left[\begin{array}{ccc}
c 1 & -s 1 & 0 \\
s 1 & c 1 & 0 \\
0 & 0 & 1
\end{array}\right]=\left[\begin{array}{lll}
0 & 0 & 1 \\
0 & 0 & 0
\end{array}\right]
$$

$$
R^{T} J^{1} W
$$

$$
\left[\begin{array}{ccc}
c 1 & s 1 & 0 \\
-s 1 & c 1 & 0 \\
0 & 0 & 1
\end{array}\right]\left[\begin{array}{ll}
0 & 0 \\
0 & 0 \\
1 & 0
\end{array}\right]=\left[\begin{array}{ll}
0 & 0 \\
0 & 0 \\
1 & 0
\end{array}\right]
$$

\section{DINÁMICA DEL ROBOT}

$$
\begin{aligned}
& \tau=\frac{d}{d t}\left[\frac{\partial \mathcal{L}}{\partial \dot{q} i}\right]-\frac{\partial \mathcal{L}}{\partial q i} \\
& \mathcal{L}=K-U \quad x 1=l 1 \cos \theta 1 \\
& \dot{x} 1=-l 1 \operatorname{sen} \theta 1 \dot{\theta} 1 \\
& U=m g h \quad y 1=l 1 \operatorname{sen} \theta 1 \\
& \dot{y} 1=l 1 \cos \theta 1 \dot{\theta} 1 \\
& U=m 1 g l 1 \operatorname{sen} \theta 1
\end{aligned}
$$

Teniendo en cuenta el enfoque analítico para el modelamiento dinámico del empuje del cuerpo ondulante, la teoría alargada del cuerpo de gran amplitud se utiliza para proporcionar un modelo dinámico para un numero de movimientos planos.

Las dinámicas de natación se calculan mediante el tratamiento de la fuerza de empuje del cuerpo y de la aleta caudal.

$$
K=\frac{1}{2} m 1 l 1^{2} \theta \dot{1}^{2}
$$




$$
\mathcal{L}=\frac{1}{2} m 1 l 1^{2} \theta \dot{1}^{2}-m 1 g l 1 \operatorname{sen} \theta 1
$$

$$
\begin{gathered}
\frac{\partial \mathcal{L}}{\partial \dot{q} 1}=m 1 l 1^{2} \dot{\theta} 1 \\
\frac{d}{d t}\left[\frac{\partial \mathcal{L}}{\partial \dot{q} 1}\right]=m 1 l 1^{2} \ddot{\theta} 1 \\
\frac{\partial \mathcal{L}}{\partial q 1}=-m 1 g l 1 \cos \theta 1 \\
\tau 1=\frac{d}{d t}\left[\frac{\partial \mathcal{L}}{\partial \dot{q} 1}\right]-\frac{\partial \mathcal{L}}{\partial q 1} \\
\tau 1=m 1 l 1^{2} \ddot{\theta} 1+m 1 g l 1 \cos \theta 1
\end{gathered}
$$

\section{DISEÑO Y CONSTRUCCIÓN DEL PROTOTIPO}

El prototipo de pez posee 2 partes importantes, la cabeza, el cuerpo y la aleta caudal, partes que fueron diseñadas en solid works de diferentes dimensiones y acopladas a las medidas de la aleta caudal. En el cuerpo se encuentra el mecanismo, tanto la parte eléctrica como la parte mecánica que es la esencia para el funcionamiento del robot, la parte electrónica está totalmente impermeabilizada para evitar daños a los componentes electrónicos.

En la cabeza del robot como se muestra en la figura 2 se va a proceder a alojar toda la parte del control, toda la electrónica y la alimentación para que el robo funcione y así aislara para que el al momento de que se sumerja no sufra riesgos la parte electrónica.
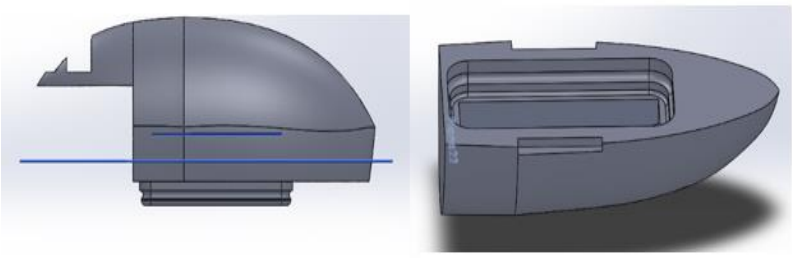

Figura 2. Diseño de la cabeza del robot 
El cuerpo servirá para poder acolar la aleta caudal y es donde se alójala el servo motor que genera el movimiento de la aleta caudal, también podemos observar en la figura 3 las aletas las que servirán de estabilidad para el pez.
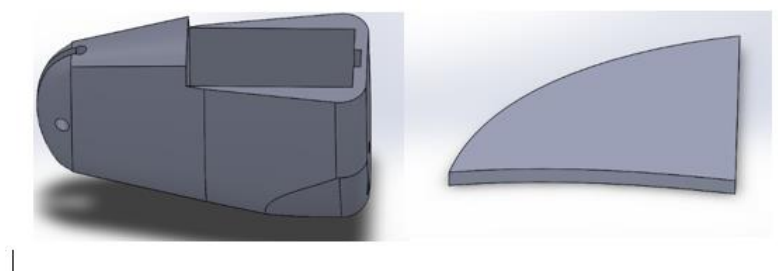

Figura 3. Cuerpo y aletas del pez

Una vez de haber diseñado por separado cada una de las partes se procede a unir todo para poder tener como resultado final el pez robot como se puede observar en la figura 4.

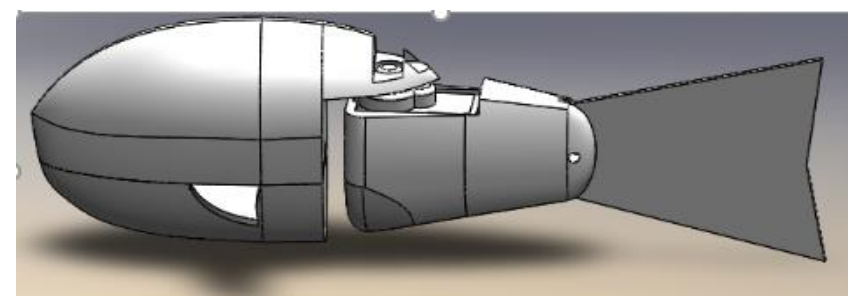

Figura 4. Prototipo del pez robot

En la figura 5 se puede observar la impresión 3d de nuestro pez diseñado y el ensamblaje total del pez ya con su aleta caudal y completamente sellado para poder ser sumergido.

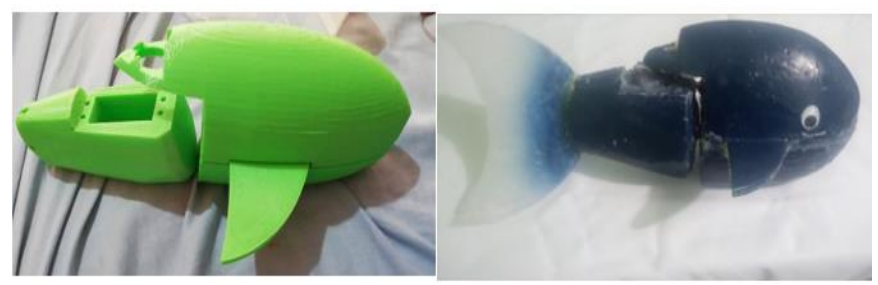

Figura 5. Impresión en 3D y ensamble del robot

Una vez que el robot pez esta listo para poder ser sumergido al agua sin que corra riesgo alguno, se procede a equilibrarlo para que sea totalmente sumergible, se procede a realizar las pruebas pertinentes para poder determinar el desplazamiento que nuestro pez realiza, este estudio se lleva a cabo mediante la utilización de un sensor IMU (inertial module unit). 


\section{Resultados}

En la figura 6 se puede observar al pez sumergido en el agua, debido a su correcta impermeabilización es posible que el pez se desplace y no sufran daño alguno sus componentes. La aleta caudal sostiene un movimiento oscilatorio, el cual permite que el robot pez se desplace en un entorno subacuático.

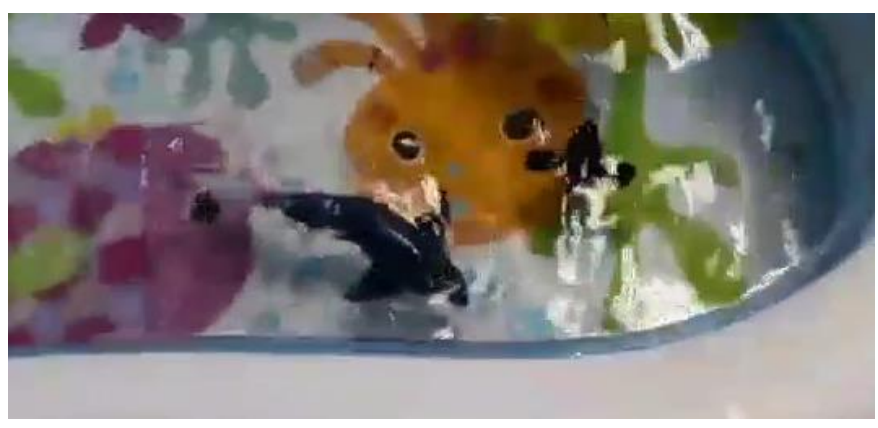

Figura 6. prueba final del pez en el agua

\section{Conclusiones}

Se diseño y construyó un pez robot con $1 \mathrm{GDL}$, su aleta caudal permite que el robot se desplace dentro de un entorno subacuático, ante lo cual fue necesario diseñar al robot para soportar la inmersión en agua dulce. Para lograr estabilidad en el desplazamiento sin que el robot empiece a girar sobre su eje de desplazamiento, se diseñaron compartimentos, dentro de los cuales es posible insertar pesas y de ésta manera estabilizar al robot, se incluyeron baterías dentro del robot las cuales dotan al prototipo de 10 minutos de autonomía en una piscina de agua dulce sin perturbaciones. Una vez construido el prototipo se sometió a pruebas de funcionalidad donde se pudo observar su correcto desempeño, se dotó al prototipo de una IMU (Inertial Module Unit), la cual permitirá en trabajos futuros analizar el comportamiento del robot en función de la variación de frecuencias de aleteo..

\section{Referencias Bibliográficas}

Drucker, E. G. 2006. “Experimental Hydrodynamics of Fish Locomotion: Functional Insights from Wake Visualization.” Integrative and Comparative Biology 42(2): $243-57$. 
Lauder, George V., and Peter G.A. Madden. 2007. "Fish Locomotion: Kinematics and Hydrodynamics of Flexible Foil-like Fins.” Experiments in Fluids 43(5): 641-53.

Rodríguez Moreno, Francy Milena, and Claudia Milena Cárdenas Martínez. 2014.

"Prototipo de Robot Submarino Con La Capacidad Del Seguimiento de

Trayectorias Mediante El Procesamiento de Imágenes."

http://repositorio.pedagogica.edu.co/handle/20.500.12209/1988 (August 7, 2019).

Sfakiotakis, M., D.M. Lane, and J.B.C. Davies. 1999. "Review of Fish Swimming Modes for Aquatic Locomotion.” IEEE Journal of Oceanic Engineering 24(2): 237-52. http://ieeexplore.ieee.org/document/757275/ (August 7, 2019).

Shoele, Kourosh, and Qiang Zhu. 2010. "Numerical Simulation of a Pectoral Fin during Labriform Swimming." The Journal of experimental biology 213(Pt 12): 2038-47. http://www.ncbi.nlm.nih.gov/pubmed/20511517 (September 15, 2019).

Villoslada, Alvaro. 2016. "Dextra."

Wang, Jianxun, Freddie Alequin-Ramos, and Xiaobo Tan. 2011. "Dynamic Modeling of Robotic Fish and Its Experimental Validation.” IEEE International Conference on Intelligent Robots and Systems: 588-94.

yu, Junzhi, Lizhong Liu, and Min Tan. 2008. "Three-Dimensional Dynamic Modelling of Robotic Fish: Simulations and Experiments." Transactions of the Institute of Measurement \& Control 30(3-4): 239-58.

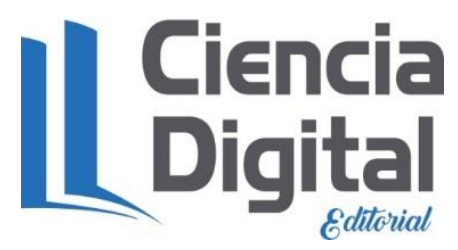




\section{PARA CITAR EL ARTÍCULO INDEXADO.}

Cabrera Aguayo, F., Santacruz Sulca, F., Bermeo Jiménez, B., \& Orozco Cazco, C. (2019). Diseño de robot pez bio inspirado impulsado mediante aleta caudal con 1 GDL. Ciencia Digital, 3(3.3), 345-355. https://doi.org/10.33262/cienciadigital.v3i3.3.826

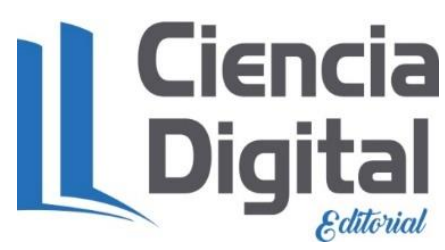

El artículo que se publica es de exclusiva responsabilidad de los autores y no necesariamente reflejan el pensamiento de la Revista Ciencia Digital.

El artículo queda en propiedad de la revista y, por tanto, su publicación parcial y/o total en otro medio tiene que ser autorizado por el director de la Revista Ciencia Digital.
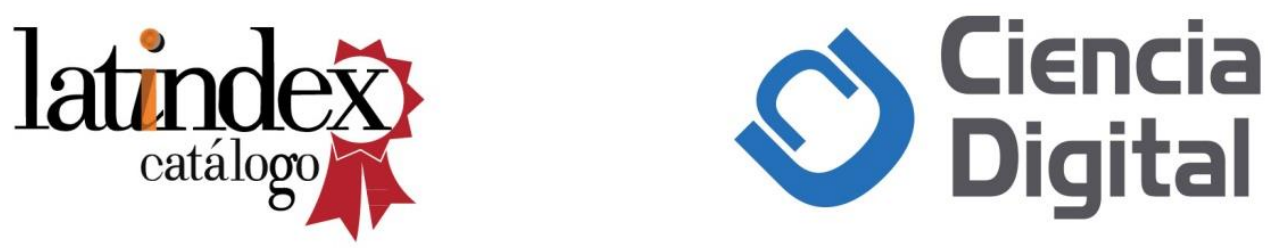